\title{
Front Tracking of Shock and Combustion Waves by an Optimal Transport Framework
}

\author{
Sabin Bhattarai Ola Marius Lysaker Dag Bjerketvedt \\ Department of Process, Energy and Environment Technology, University of South-Eastern Norway, Norway, \\ \{sabinbhattarai1994@gmail.no, marius.lysaker@usn.no, dagbj@usn.no\}
}

\begin{abstract}
This paper presents a framework for front tracking of shock waves from high-speed video recordings. The video was corrupted with noise and artifact that made front tracking a challenging task. To our knowledge, the implementation of Unnormalized Optimal Transport for determination of velocity is novel.
\end{abstract}

An Unnormalized Optimal Transport framework was implemented in Python to determine the route of propagation from the recorded high-speed video. The obtained route was further computed for front tracking by two methods; Divergence and Transport method. These methods were investigated with both synthetic images and the recorded high-speed video frames. For both, the Transport method provided better results than the Divergence method. Therefore, the shock wave velocity and the shock angles were calculated from Unnormalized Optimal Transport combined with the Transport method. Preliminary results indicate that our findings are in good agreement with sensor-based measurements.

This framework verified that the triple point height is increasing, and the oblique shockwave moves faster than the normal shock wave.

Keywords: unnormalized optimal transport, front tracking, image processing

\section{Introduction}

Combustion and shock waves propagate at high speed which makes challenging to find the velocity and shape of the flame and shock wave. In lab experiments, stateof-the-art has been to use of sensors like pressure transducers to estimate velocities. One drawback of that technique is the limited number of sensors placed inside a test rig. An alternative to these sensor-based measurements is to use a high-speed camera whereon the position, shape, and velocity can be found from the recorded high-speed images. Video recording can be analyzed frame by frame to track wave. Even though humans can comfortably identify and track such waves in a video, it is challenging to design robust algorithms for automatic tracking. Also, the presence of noise and artifact made the task more challenging. Although various algorithms, such as Level set (Osher and Sethian, 1988), Splines (Maharjan et al., 2019), etc. have been proposed, we are evaluating unnormalized optimal transport for tracking waves.

The French mathematician Gaspard Monge pioneered on optimal transport in 1781. Later, the Russian Mathematician Leonid Vitaliyevich Kantorovich stated and proved the problem through the additional functional tools known as duality theorem. Monge explained optimal transport as distance covered to transfer a pile of sand from one location to another with minimum transport cost. If this cost is a function of distance, the distance from optimal transport is called the Kantorovich-Rubinstein distance or Wasserstein distance (Villani, 2008). Optimal transport gained popularity in inverse problems and machine learning.

The above classical approach from Monge and Kantrovich is in the normalized density space, i.e., the masses of two density functions or histogram is equal. Gangbo et al. (2019) have described a formulation in which the masses of two density functions are unequal, refer to it as unnormalized or unbalanced optimal transport. With this approach, it is possible to examine two images with different intensities. Therefore, the high-speed video was analyzed with unnormalized optimal transport.

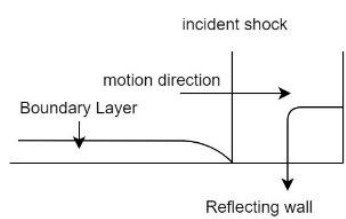

(a)

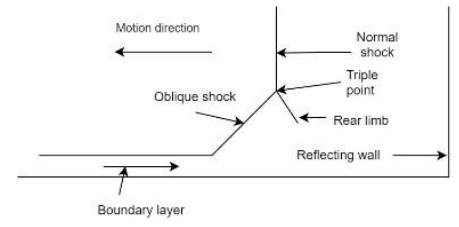

(b)
Figure 1. Schematic diagram of bifurcated shock wave a) Incident shock wave b) Reflected shock wave

The high-speed video images provided the shock wave position. A shock wave is a thin compression wave across which the gas changes its thermodynamic properties such as density and pressure (Bjerketvedt et al., 1997). When a normal incident shock wave hits the end wall, it is reflected. The reflected shock wave is bifurcated due to the boundary layer. Figure 1 shows the bifurcation of the reflected shock wave. This occurs if stagnation pressure in the boundary layer is less than 
the pressure behind the normal reflected shock wave (Damazo et al., 2012).

\section{Methods}

The Test rig of the experiment is described in Siljan et al. (2017). The high-speed video from this experiment was analyzed with methods below:

\subsection{Image conversion}

Image conversion is pre-processing for optimal transport. The input for optimal transport is in densities space. The pixel values were converted into densities and must be non-negative values. The obtained images were resizing into the same scale.

\subsection{Unnormalized Optimal Transport}

The unnormalized optimal transport was implemented to determine the route of the traveling wave. $L^{1}$-norm was used as a distance function in the unnormalized optimal transport as it requires less computation time and is simple for implementation compared to the $L^{2}$ norm (Li et al., 2018). So, implemented optimal transport is $L^{1}$ - unnormalized optimal transport. The algorithm for $L^{1}$ - unnormalized optimal transport is mention below (Osher, 2019):

$$
\begin{gathered}
m^{k+1}=\frac{m^{k}+\tau_{1} \cdot \nabla \Phi}{\left|m^{k}+\tau_{1} \cdot \nabla \Phi\right|}\left(\left|m^{k}+\tau_{1} \cdot \nabla \Phi\right|-\tau_{1}\right) \\
\Phi^{k+1}=\Phi^{k}+\tau_{2}\left(\nabla \cdot m^{k+1}-\nabla \cdot m^{k}+\mu^{1}-\mu^{0}\right. \\
\quad+c) .
\end{gathered}
$$

Where,

$$
c=\frac{1}{\text { row } \times \text { col }}\left(\text { sum of } \mu^{1}-\text { sum of } \mu^{0}\right) \text {, }
$$$$
\text { row }=\text { number of rows of } \mu^{1} \text { or } \mu^{0} \text {, }
$$

col $=$ number of columns of $\mu^{1}$ or $\mu^{0}$.

Here, $\mu^{1}$ and $\mu^{0}$ are input images that display the movement of an object. $\tau_{1}$ and $\tau_{2}$ are step sizes. $c$ is constant for compensation of change of intensities between images.

The solution obtained is $m$ that is a vector, and $\Phi$ is the Lagrange multiplier. The $m$ consists of two components represented as $m_{x}$ and $m_{y}$. It was found that $m_{x}$ and $m_{y}$ are responsible for the horizontal movement and vertical movement, respectively.

Initially, the value of $m$ and $\Phi$ is zero. Iteration continued until the solution converged. The iteration time depends upon grid size or pixel size of images, i.e., smaller grid sizes mean more data that increases the execution time. So, multilevel unnormalized transport is implemented for faster calculation (Liu et al., 2019).

A detailed study of this method can be found in (Gangbo et al., 2019; Chambolle and Pock, 2011; Li et al., 2018).

\subsection{Post-processing}

The outcomes from unnormalized optimal transport are used as input to the front tracking. This process is accomplished by using the Transport and Divergence method.

\subsubsection{Transport method}

The resultant from these two components are calculated using the formula below:

$$
\text { resultm }=\sqrt[2]{m_{x}^{2}+m_{y}^{2}}
$$

The obtained result has proceeded for edge detection after Total Variation (TV) denoising. TV denoising is used to filter resultm (from above equation) as it preserves the edge better (Chambolle and Pock, 2011). Edge detection is accomplished by the Sobel Operator (Bovik, 2009). The maximum of each row from the initial column to the middle of the unnormalized optimal transport route is used as a tracked front from edge detected images.

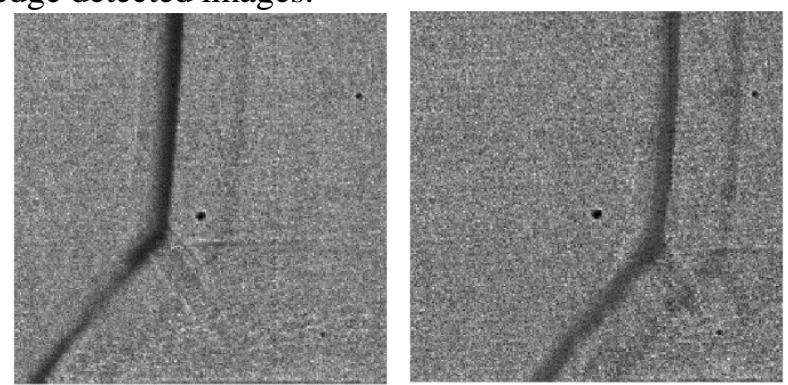

Figure 2. Images presenting the propagation of shock wave.

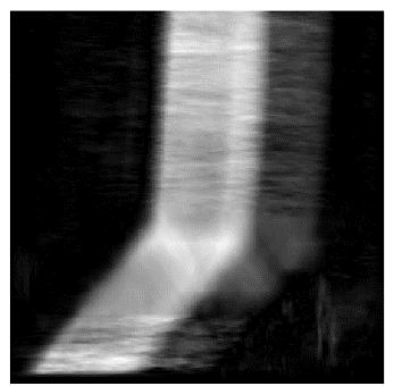

(a)

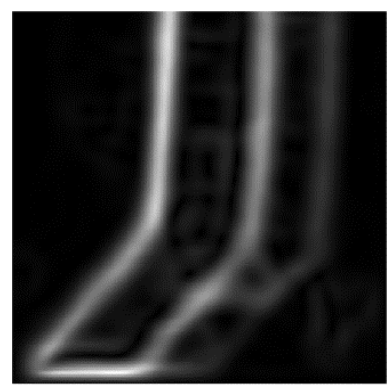

(b)
Figure 3. a) Unnormalized optimal transport outcome b) Edge detection of a)

Consider Figure 2 as inputs for unnormalized optimal transport to demonstrate this method. The obtained solution $m$ is formulated with (3) is in Figure 3(a). This figure undergoes TV denoising due to noise caused by turbulence from the shock wave movement. The denoised result is used for edge detection with the Sobel operator in Figure 3(b). The data extracted as maximum from the left edge from Figure 3(b) as the right edge is disturbed by the rear limb in Figure 1(b). The tracked front is in Figure 4. 


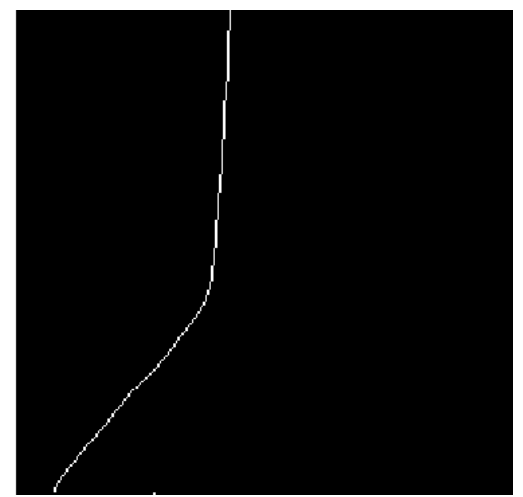

Figure 4. Tracked front from Figure 2

\subsubsection{Divergence method}

The dynamic constraint for unnormalized optimal transport is integrated with the time variable (Gangbo et al., 2019). We obtain the following equation:

$$
\nabla \cdot m=\mu_{0}-\mu_{1}+c \text {. }
$$

Here, the initial image $\left(\boldsymbol{\mu}_{\mathbf{0}}\right)$ refers to an object's initial position in an image, and the final image $\left(\boldsymbol{\mu}_{1}\right)$ refers to the moved position object in the next one during movement. The divergence of $\mathrm{m}$ incorporates initial image and final images, and $\boldsymbol{c}$ is constant to deal with intensities change. If the final images $\left(\boldsymbol{\mu}_{\boldsymbol{1}}\right)$ is zero, then the remaining will only be initial images and difference between initial and final images intensities, i.e., $\boldsymbol{\nabla} \cdot$ $\boldsymbol{m}=\boldsymbol{\mu}_{\mathbf{0}}+\boldsymbol{c}$. So, image from $\boldsymbol{\nabla} \cdot \boldsymbol{m}$ is used for front tracking. This method is illustrated using the same example as above.

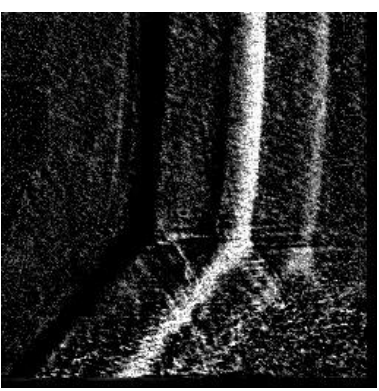

(a)

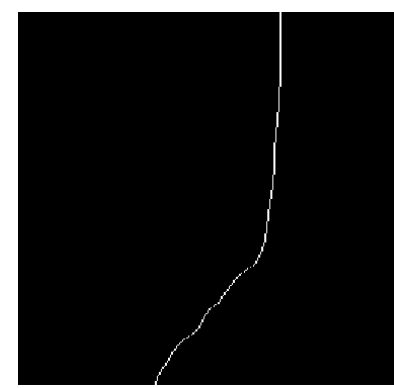

(b)
Figure 5. a) Divergence of unnormalized optimal transport result(m) b) Tracked front from Figure 5(a)

Figure 5(a) is the divergence of $m$ with $\mu_{1}=0$. The obtained image is similar to the second image in Figure 2 , but the shock wave is more distinct. The maximum of each row is extracted and presented in Figure 5(b).

\section{Result and Discussion}

\subsection{Comparing methods}

The unnormalized optimal transport outcome was further analyzed to calculate velocity by using the two methods mentioned above. These two methods are compared with noisy and noise-free synthetic images and experimental video images below.

\subsubsection{Synthetic images}

The developed series of images displays a slender's horizontal movement in Figure 6 as the shock wave movement in the video.
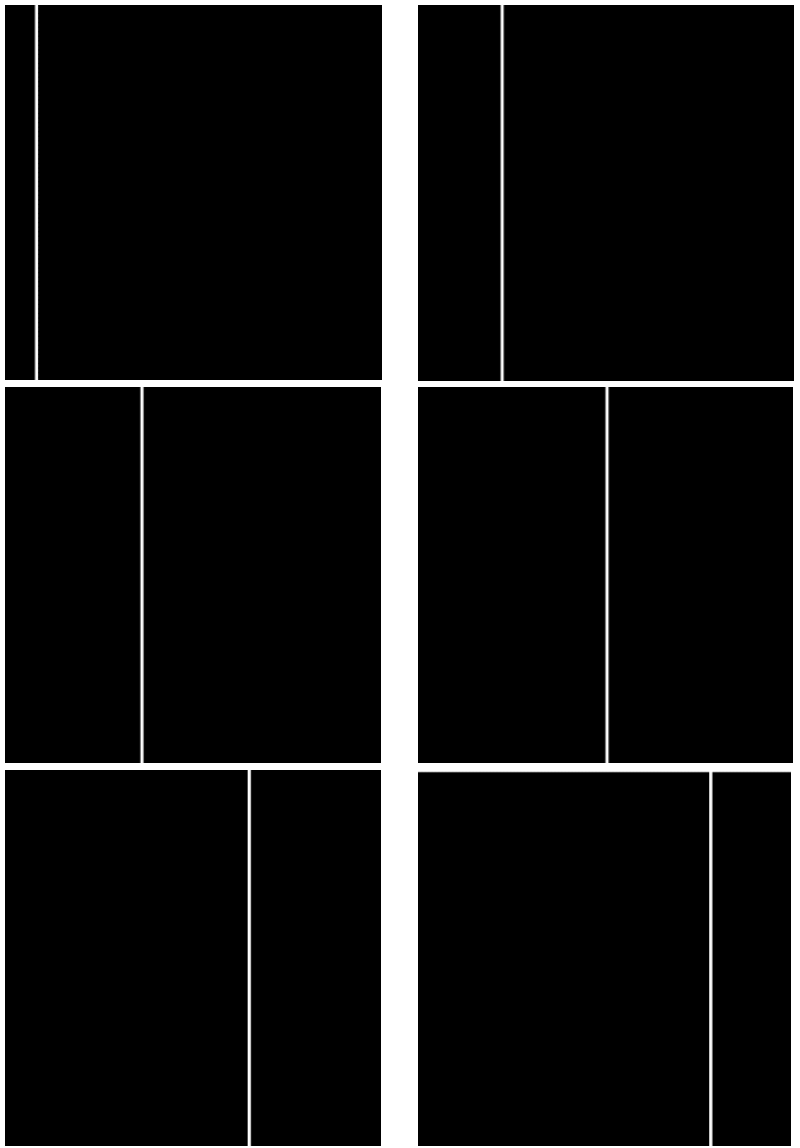

Figure 6. Series of the synthetic image showing slight movement.

The transport and divergence method applied to the solution of unnormalized optimal transport shown in Figure 6. The results from these methods are in Figure 7(a) and Figure 7(b), respectively. The transport method results in Figure 7(a) tracked slender more accurately than the divergence method results in Figure 7(b). Even though the transport method shows a better result, it cannot find the first row of each slender's position in images.

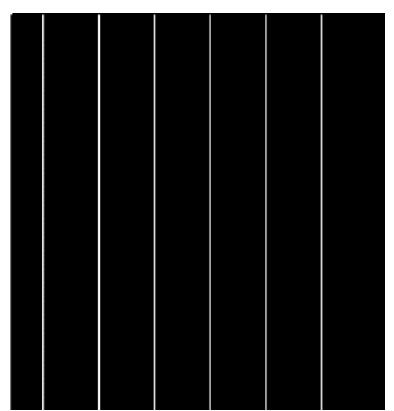

(a)

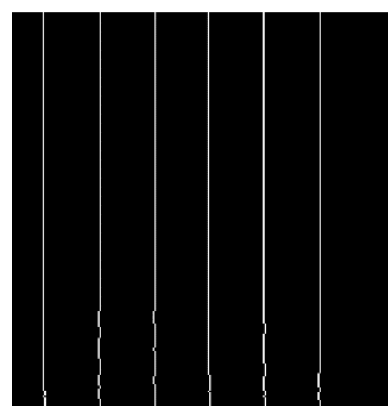

(b)
Figure 7. Tracked slender from noise-free images a) Transport method b) Divergence method 
The images from Figure 6 are added with noise. Noise is a normal distribution whose mean is zero and the standard deviation is 10 . The remaining assumption is of these images are equivalent to noise-free synthetic images. The slender is tracked based on the above mention methods, illustrated in Figure 8. The Transport method cannot determine the front of the first and last row, but the remaining portion of the tracked slender shown in Figure 8(a) is exact as input. The determined slender by divergence method is zigzag shown in Figure $8(\mathrm{~b})$. The transport method is better than the divergence method displayed in Figure 8.

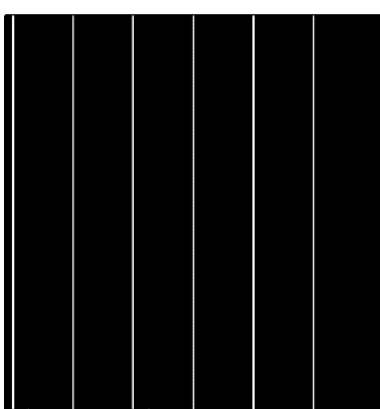

(a)

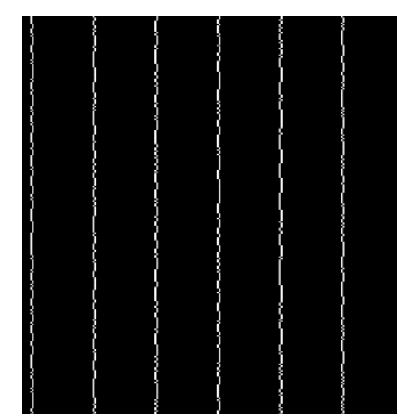

(b)
Figure 8. Tracked slender from noisy images a) Transport method b) Divergence method.

\subsubsection{High-speed video}

The high-speed video from the experiment includes a series of images displaying the shock wave movement, i.e., incident shock and reflected shock wave. The video was broken into images, and a single image refers to the frame. Images from frame number 80 to frame number 175 of video are input for analysis. The unnormalized optimal transport was implemented at an interval of 5 frames. The outcome of unnormalized optimal transport is the data for tracking front using the divergence and transport method illustrated in Figure 9.

Visually, the front tracking with the transport method is better than the divergence method from Figure 9. Although the tracked fronts are bend at the bottom with the transport method, it has a smoother transition between tracked points for wave than the divergence method. The problem might be the data extraction procedure in the divergence method. So, the results are favoring the transport method. Hence, we are proceeding with the transport method.

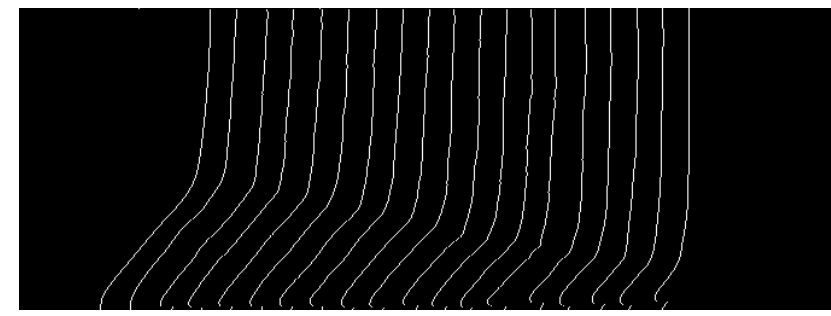

(a)

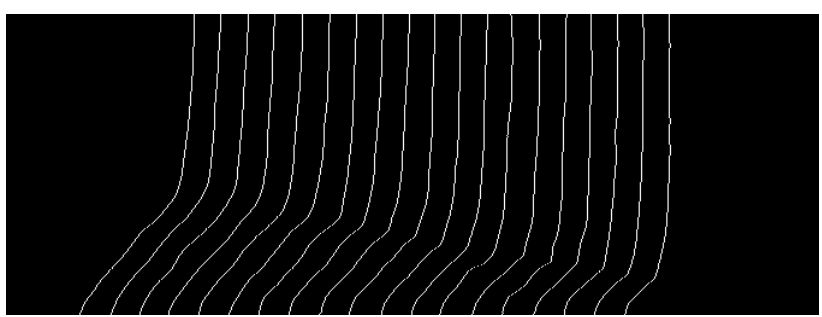

(b)

Figure 9. Tracked front from reflected shock wave with unnormalized optimal transport at an interval of 5 frames a) Transport method b) Divergence method.

The unnormalized optimal transport was implemented at an interval of 3 frames, and shock wave tracking accomplished using the transport method as illustrated in Figure 10. In comparison between Figure 9(a) and Figure 10, it was found that unnormalized optimal transport at the interval of 5 frames is providing better results than at the intervals of 3 frames. The further analysis uses the unnormalized optimal transport at the intervals of 5 frames and the transport method.

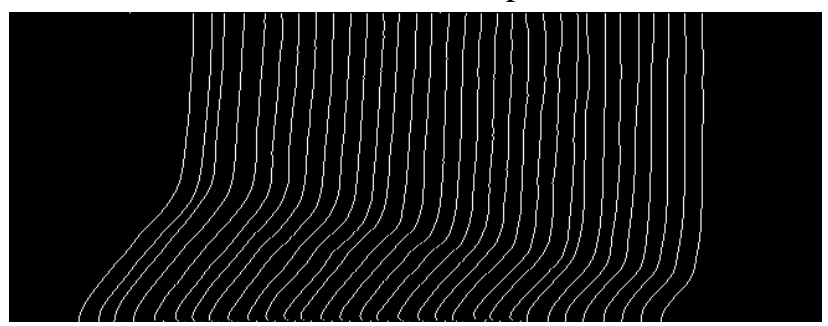

Figure 10. Tracked front from reflected shock wave with unnormalized optimal transport at an interval of 3 frames and Transport method

\subsection{Velocity Determination}

Assume two fronts shown in Figure 11 for the calculation of the velocity of the shockwave. Let us denote $d_{1}(x, y)$ as a pixel position on the front $(f)$ and $d_{2}(x, y)$ as a pixel position on the consecutive front $(f+1)$ shown in Figure 9. Here, $x$ and $y$ represent row and column in images as per Python.

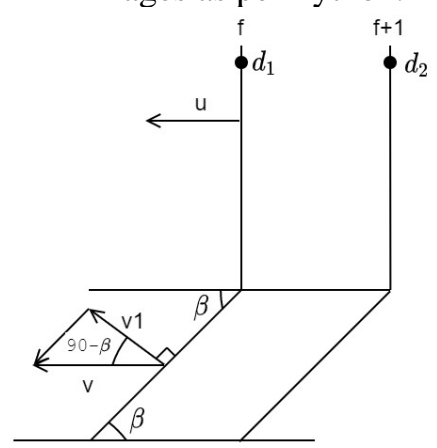

Figure 11. Schematic sketch of two consecutive shock waves.

The distance between the fronts is calculated as $d=$ $\left|d_{1}-d_{2}\right|$. The obtained difference undergoes mathematical operation of the scaling factor and the high-speed camera frame rate for computation of actual 
velocity. The scaling factor for an image from the highspeed video is $1 \mathrm{~mm}=9.528 \pm 0.079$ pixel. The frame rate of the high-speed camera is 500000 frames per second (FPS). The velocity is calculated with (5) for images at the interval of 5 frames.

$$
v=\frac{d}{5} \times \frac{500000}{9528}\left[\frac{\mathrm{m}}{\mathrm{s}}\right]
$$

The velocity $(u)$ represented the normal shock wave, and $(v)$ represented the oblique shock wave. The oblique shock wave is tilted with angle $(\beta)$, which is known as the shock angle. The oblique shock wave velocity is divided into normal and parallel components. The normal component of velocity $(v)$ is represented as $v 1$.

$$
v 1=v \cdot \cos (90-\beta)=v \cdot \sin \beta
$$

The 20 points from the upper portion of each tracked fronts from row number 1 to row number 21 were used to calculate the normal shock wave's average velocity. Similarly, 20 points starting from row number 195 to 215 are extracted to calculate the oblique shock wave's average velocity. The normal velocity for the oblique shock wave can only be calculated after the calculation of shock angles.

Figure 12, oblique shock wave crossed by the upper and lower boundary, is data for estimating shock angles. This figure illustrated that the upper and lower boundary crosses tracked oblique shock. The lower boundary is straight, but the upper boundary is not because the oblique shock length increases (the elevation of the triple point is increasing) as shock wave propagates. The data is extracted from the upper to lower boundary for each front separately. The extracted data trend is a straight line, and the straight line equation is fitted with extracted data by the leastsquares method. The inclination of the obtained straight-line reference to the bottom side of images is shock angles. These angles and oblique shock wave velocities are required to calculate the oblique shock wave's normal velocity with (6).

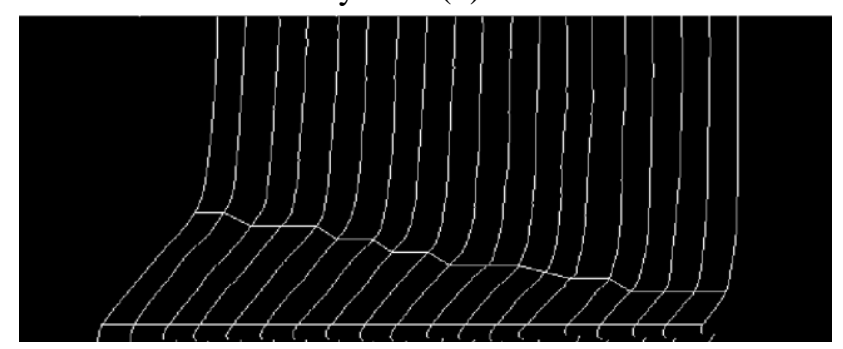

Figure 12. The region selected for calculation of shock angles from tracked fronts with transport method

The velocities calculated above are presented in Figure 13. The $\mathrm{x}$-axis in this figure is the distance from the shock reflected wall. It is estimated from 10 points from upper tracked fronts.

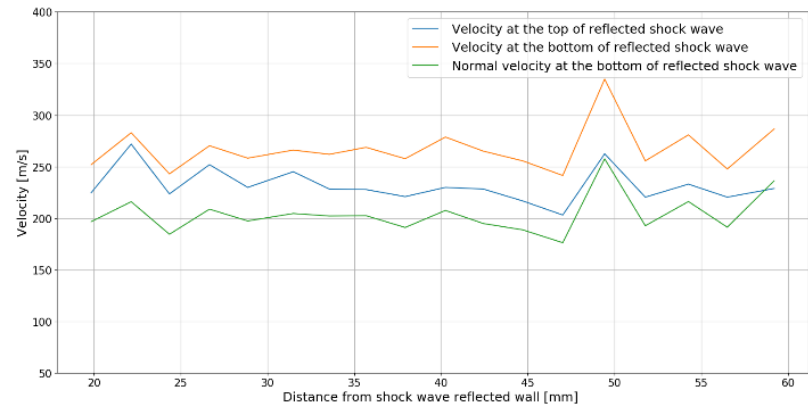

Figure 13. Average velocities of normal and oblique shockwave, and normal component of an oblique shock wave with unnormalized optimal transport with 5 frames and transport method.

Figure 13 displays that the oblique shock wave moves faster than a normal shock wave as in the high-speed video. This result is obtained without filtering inputs for unnormalized optimal transport.

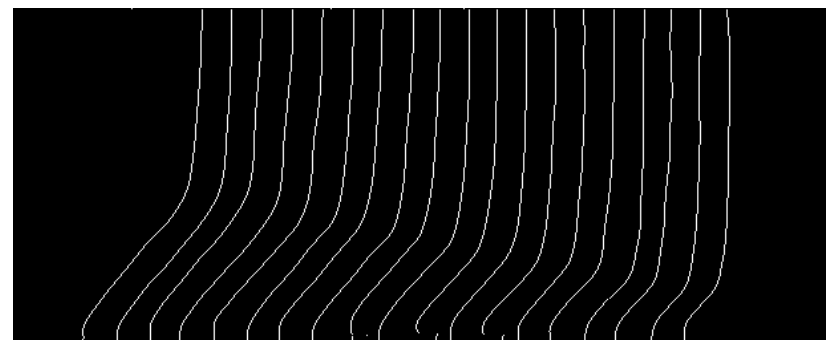

Figure 14. Tracked fronts from reflected shock wave with unnormalized optimal transport at an interval of 5 frames in which series of operations performed in input images for denoising and transport method.

The images from the given high-speed video are noisy. Hence, images are denoised before the implementation of unnormalized optimal transport. In this process, background subtraction, morphological operation (closing), and TV denoising proceed in a sequence. Firstly, the first image from the given video subtracted from noisy images displaying shock propagation. Successively, the morphological operation (closing) is performed in subtracted images as it fills a tiny gap near the edge (Maharjan et al., 2019) followed by TV denoising. The obtained images are input for unnormalized optimal transport followed by the transport method, and the result in Figure 14.

Figure 14 exhibits a smoother curve of shock wave than in Figure 9(a) but there is not much change in tracked front with filtered and unfiltered inputs for unnormalized optimal transport. The velocities calculated for filter images from (5) and (6) are in Figure 15. Figure 13 shows higher oscillation in the velocities curve than in Figure 15 as denoising operations in input images. Although result from unfiltered images produces high oscillation velocities curves, the trend for normal shock wave velocity, oblique shock wave velocity, and normal velocity for filter and unfiltered input of unnormalized optimal transport similar. 


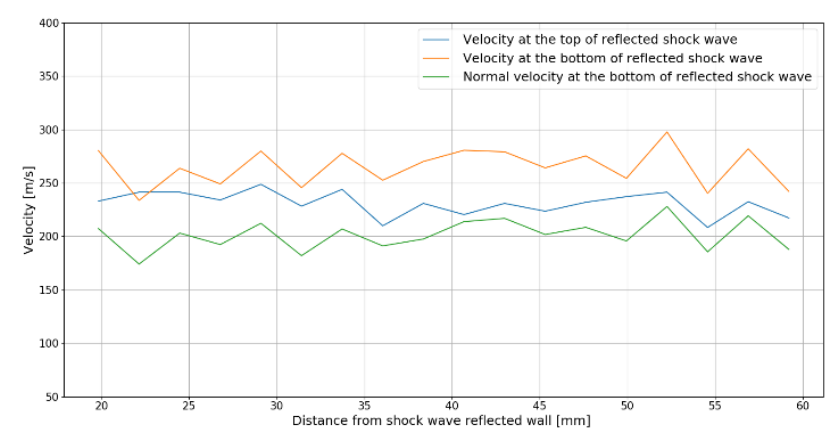

Figure 15. Average velocities of the normal and oblique shock wave, and normal component of the oblique shock wave with unnormalized optimal transport at an interval of 5 frames in which series of operation performs in input images denoising and transport method.

Figure 16 demonstrates that trends of shock angles of unfiltered and filtered images are slightly different but fluctuate around $50^{\circ}$. The average shock angles of unfiltered and filtered images are $49.7^{\circ}$ and $49.4^{\circ}$, respectively.

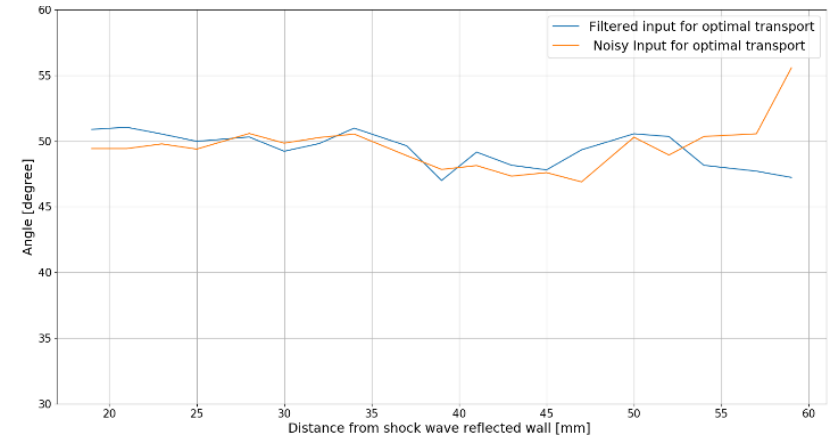

Figure 16. Oblique shock angle from unnormalized optimal transport at an interval of 5 frames and transport method.

If a tracked front deviate from one pixel, then it results in an error of $11 \mathrm{~m} / \mathrm{s}$ considering images as an input interval of 5 frames.

\subsection{Comparison with combustion, process safety, and explosion research group}

Researches had conducted simulation for computation of the velocity with the same high-speed video. The solution from pattern matching and segmented regression (Siljan et al., 2017), dynamic template matching (Maharjan, 2019), and snake model with gradient vector fields and watershed segmentation (Maharjan et al., 2019) and sensor-based calculations are in Table 1. The mentioned frameworks and experimental results for oblique shock wave velocities are within $5 \mathrm{~m} / \mathrm{s}$ deviation but above $10 \mathrm{~m} / \mathrm{s}$ deviation for normal shock wave velocities. The pressure sensors are presented at the bottom, so the normal shock wave velocities are calculated from the oblique shock wave pressure.

Similarly, the difference in shock angle between simulated results and experimental value is less than $3^{\circ}$. The results of unnormalized optimal transport and other frameworks are within $5 \%$ fluctuation.

Table 1. Comparison between results from different methods for reflected shock wave

\begin{tabular}{|c|c|c|c|c|}
\hline S.N & $\begin{array}{l}\text { Descriptio } \\
\mathrm{n}\end{array}$ & $\begin{array}{l}\text { Average } \\
\text { velocity } \\
\text { of normal } \\
\text { shock } \\
\text { wave }[\mathrm{m} / \mathrm{s} \\
\text { ] }\end{array}$ & $\begin{array}{l}\text { Average } \\
\text { velocity } \\
\text { of oblique } \\
\text { shock } \\
\text { wave[m/s } \\
\text { ] }\end{array}$ & $\begin{array}{l}\text { Averag } \\
\text { e shock } \\
\text { angle }\end{array}$ \\
\hline 1. & $\begin{array}{l}\text { Result } \\
\text { from the } \\
\text { pressure } \\
\text { transducer } \\
\text { (Maharjan } \\
\text { et al., } \\
\text { 2019) }\end{array}$ & 216 & 266 & $48^{\circ}$ \\
\hline 2. & $\begin{array}{l}\text { Result } \\
\text { from } \\
\text { template } \\
\text { matching } \\
\text { (Siljan et } \\
\text { al., 2017) }\end{array}$ & 230 & 262 & $50^{\circ}$ \\
\hline 3. & $\begin{array}{l}\text { Result } \\
\text { from } \\
\text { snakes } \\
\text { model } \\
\text { (Maharjan } \\
\text { et al., } \\
\text { 2019) }\end{array}$ & 227 & 267 & $48.9^{\circ}$ \\
\hline 4. & $\begin{array}{l}\text { Result of } \\
\text { optimal } \\
\text { transport } \\
\text { with filter } \\
\text { images } \\
\text { (here) }\end{array}$ & 230.8 & 265 & $49.4^{\circ}$ \\
\hline 5. & $\begin{array}{l}\text { Result of } \\
\text { optimal } \\
\text { transport } \\
\text { with } \\
\text { unfiltered } \\
\text { images } \\
\text { (here) }\end{array}$ & 231.5 & 267 & $49.7^{\circ}$ \\
\hline
\end{tabular}

\section{Conclusion}

The above section demonstrated that the present framework can estimate velocities and shock angles from experimental results and synthetic images from other algorithms. The velocity is obtained from a series 
of images representing an object's motion with unnormalized optimal transport.

The transport method provided better front tracking of unnormalized optimal transport results than the divergence method. Moreover, filtering the input to the unnormalized optimal transport had only minor impact on the results.

\section{References}

A. Bovik. The Essential Guide to Image Processing. Elsevier Science \& Technology, 2009.

Url:http://ebookcentral.proquest.com/lib/ucsnebooks/detail.action?docID=452947.

A. Chambolle, and T. Pock. A First-Order Primal-Dual Algorithm for Convex Problems with Applications to Imaging. Journal of Mathematical Imaging and Vision, 40(1):120-145, 2011. doi: 10.1007/s10851-010-0251-1.

W. Gangbo, W. Li, S. Osher, and M. Puthawala. Unnormalized Optimal Transport. Journal of Computational Physics, 399, 108940, 2019. doi: 10.1016/j.jcp.2019.108940.

W. Li, E. K. Ryu, S. Osher, W. Yin, and W. Gangbo. Parallel Method for Earth Mover's Distance. Journal of Scientific Computing, 75(1): 182-197, 2018. doi: 10.1007/s10915017-0529-1.

J. Liu, W. Yin, W. Li, and Y. T. Chow. Multilevel Optimal Transport: A Fast Approximation of Wasserstein-1 distances, 2019. doi: http://arxiv.org/abs/1810.00118.

S. Maharjan, D. Bjerketvedt, and O. M. Lysaker. An Image Processing Framework for Automatic Tracking of Wave Fronts and Estimation of Wave Front Velocity for a Gas Experiment. In L. Chen, B. Ben Amor, \& F. Ghorbel (Eds.), Representations, Analysis and Recognition of Shape and Motion from Imaging Data: 45-55, 2019. doi: 10.1007/978-3-030-19816-9_4.

S. Osher. Unnormalized Optimal Transport.,2019. doi: https://ipam.wistia.com/medias/2015gis083?wtime $=0$.

S. Osher and J.A. Sethian. Fronts propagating with curvature-dependent speed: Algorithms based on Hamilton-Jacobi formulations. Journal of Computational Physics, 79(1): 12-49, 1988. doi: 10.1016/00219991(88)90002-2.

E. Siljan, M. Lysaker and S. Maharjan. Wave Front Tracking using Template Matching and Segmented Regression: 326-331, 2017. doi: 10.3384/ecp17138326. 